\title{
Full-Profile X-Ray Phase Analysis of the Composition of Aluminum Electrolyte on the Basis of a Multipopulation Evolutionary Algorithm and Elemental Analysis Data
}

\author{
Petr S. Dubinin*a, Alexandr N. Zaloga ${ }^{a}$, \\ Sergey V. Burakov ${ }^{\mathrm{b}}$, Konstantin A. Gusev', \\ Evgeniy S. Andryushchenko ${ }^{a}$, Igor S. Yakimov', \\ Oksana E. Bezrukova ${ }^{a}$ and Alexandr S. Samoilo ${ }^{a}$ \\ ${ }^{a}$ Siberian Federal University \\ 79 Svobodny, Krasnoyarsk, 660041 Russia \\ ${ }^{b}$ Reshetnev Siberian State University of Science and Technology \\ 31 Krasnoyarsky Rabochy, Krasnoyarsk, 660037, Russia
}

Received 28.03.2018, received in revised form 03.04.2018, accepted 12.05.2018

\begin{abstract}
We developed a method of self-configurable multipopulational parallel genetic algorithm (SGA) and successfully applied it to a standardless automatic full-profile X-ray phase analysis of aluminum bath samples. The method spares a user-analyst from working on the empirical choice of the genetic algorithm settings and shows the efficiency of the automatic QPA by the Rietveld method under the control of the SGA, as well as the possibility of using it for express monitoring of the bath composition in aluminum production.
\end{abstract}

Keywords: genetic algorithms, self-configuration, X-ray powder diffraction, full-profile analysis, Rietveld method, quantitative $X$-ray phase analysis, aluminum electrolyte.

Citation: Dubinin P.S., Zaloga A.N., Burakov S.V., Gusev K.A., Andryushchenko E.S., Yakimov I.S., Bezrukova O.E., Samoilo A.S. Full-profile x-ray phase analysis of the composition of aluminum electrolyte on the basis of a multipopulation evolutionary algorithm and elemental analysis data, J. Sib. Fed. Univ. Chem., 2018, 11(2), 262-272. DOI: 10.17516/1998-28360073 .

(C) Siberian Federal University. All rights reserved

* Corresponding author E-mail address: dubinin-2005@yandex.ru 


\title{
Полнопрофильный рентгенофазовый
}

\section{анализ состава электролита алюминиевого производства \\ на основе мультипопуляционного эволюционного алгоритма \\ и данных элементного анализа}

\author{
П.С. Дубинин ${ }^{\mathrm{a}}$, А.Н. Залога ${ }^{\mathrm{a}}$, \\ С.В. Бураков ${ }^{0}$, К.А. Гусев ${ }^{\tilde{0}}$, Е.С. Андрющенко ${ }^{a}$, \\ И.С. Якимов ${ }^{a}$, О.Е. Безрукова ${ }^{a}$, А.С. Самойло ${ }^{a}$ \\ ${ }^{a}$ Сибирский федеральньий университет \\ Россия, 660041, Красноярск, пр. Свободнылй, 79 \\ ${ }^{6}$ Сибирский государственный университет науки \\ и технологий имени М.Ф. Решетнева \\ Россия, 660037, Красноярск, пр. Красноярский рабочий, 31
}

\begin{abstract}
Разработан метод количественного рентгенофазового анализа (КРФА) на основе самоконфигурируемого мультипопуляиионного параллельного генетического алгоритма (СГА), который успешно применен для бесстандартного автоматического полнопрофильного анализа образиов электролита алюминиевых электролизеров. Метод позволяет избежать работь по эмпирическому выбору настроек генетического алгоритма и показывает достаточную точность автоматического КРФА по методу Ритвельда под управлением СГА (полученные значения криолитового отношения соответствуют аттестованным значениям с точностью, характеризуемой стандартным отклонением всего 0,035 ед. КО), а также возможность его использования для оперативного контроля состава электролита на алюминиевом производстве.
\end{abstract}

Ключевые слова: генетические алгоритмы, самоконфигурация, рентгеновская порошковая дифракция, метод Ритвельда, количественный рентгенофазовый анализ, электролит алюминиевого производства.

\section{Введение}

В настоящее время алюминий получают в электролизных ваннах разложением глинозема $\left(\mathrm{Al}_{2} \mathrm{O}_{3}\right)$, растворенного в расплаве фторидов при температуре около $950{ }^{\circ} \mathrm{C}$ [1]. Основным компонентом расплава является натриевый криолит $\left(\mathrm{Na}_{3} \mathrm{AlF} \mathrm{F}_{6}\right)$, к которому для улучшения технологических характеристик добавляют фторид алюминия $\left(A l F_{3}\right)$ и некоторые фториды щелочных и щелочноземельных металлов, чаще всего это фторид кальция $\left(\mathrm{CaF}_{2}\right)$ и фторид магния $(\mathrm{MgF})$. В результате введения добавок изменяются важные для технологии электролиза свойства расплавов: электропроводность, плотность, температура ликвидуса, растворимость глинозема, давление паров над расплавом, а также коррозионное воздействие на материалы электродов, футеровку и другие элементы конструкции электролизера. В процессе работы электролизная ванна постоянно испытывает колебания и отклонения от оптимального химического состава.

$$
-263-
$$


Поддержание постоянного химического состава ванны является важнейшим элементом технологии производства алюминия. Поскольку даже в расплаве основные элементы связаны в определенные химические формы, для характеристики состава расплава вблизи оптимального состава не требуется знания всех концентраций, а достаточно одной величины.

Таким основным технологическим параметром, характеризующим химический состав электролизной ванны, выступает криолитовое отношение $(K O)$ - интегральная величина, выражающая величину мольного отношения фторида натрия к фториду алюминия или отношение их масс:

$$
K O=\frac{\mathrm{C}(\mathrm{NaF}, \text { мол.\% })}{\mathrm{C}\left(\mathrm{AlF}_{3}, \text { мол.\% } \%\right)}=2 \cdot \frac{\mathrm{C}(\mathrm{NaF}, \text { масс.\% })}{\mathrm{C}\left(\mathrm{AlF}_{3}, \text { масс.\% }\right)},
$$

где $\mathrm{C}$ - содержания фторидов натрия и алюминия в молярных или массовых процентах.

Дополнительно, наряду с $K O$, контролируются концентрации добавок фторидов. Технологический экспресс-анализ состава электролита производится количественным рентгенофазовым методом (КРФА). Анализ проводится в лабораторных условиях на отобранных из ванн и охлажденных пробах по измеряемым на дифрактометрах интенсивностям аналитических линий кристаллических фаз. Необходимая частота контроля ванны - раз в 2-3 дня, точность контроля $K O \sim 0,04$, точность контроля фторидов $\sim 10 \%$ (отн.), время на анализ одной пробы несколько минут.

Охлажденные пробы электролита российских алюминиевых заводов имеют достаточно сложный фазовый состав, приведенный в табл. 1.

В промышленных материаловедческих лабораториях для КРФА обычно используются методики, основанные на методе внешнего эталона, т.е. на использовании калибровочных графиков, построенных с помощью набора стандартных образцов - известного количественного фазового состава. Данный метод обладает рядом преимуществ, среди которых высокая точность и автоматизация. Однако серьезным недостатком метода внешнего эталона является необходимость синтеза адекватных по химическому фазовому составу и микрокристаллическим

Таблица 1. Фазы проб охлажденного электролита российских алюминиевых заводов

Table 1 . The electolyte at Russian aluminum smelters

\begin{tabular}{|c|l|c|c|c|}
\hline № & \multicolumn{1}{|c|}{ Фаза } & Хим. формула & Содержание $(\%$ масс.) & Область КО \\
\hline 1 & Криолит & $\mathrm{Na}_{3} \mathrm{AlF}_{6}$ & $0 \div 90$ & $>1.67$ \\
\hline 2 & Хиолит & $\mathrm{Na}_{5} \mathrm{Al}_{3} \mathrm{~F}_{14}$ & $0 \div 85$ & $<3.0$ \\
\hline 3 & Фторид натрия & $\mathrm{NaF}$ & $0 \div 5$ & $>3.0$ \\
\hline 4 & Са-криолит 1 & $\mathrm{NaCaAlF}_{6}$ & $0 \div 15$ & $<3.0$ \\
\hline 5 & Са-криолит 2 & $\mathrm{Na}_{2} \mathrm{Ca}_{3} \mathrm{Al}_{2} F_{14}$ & $0 \div 20$ & $>2.45$ \\
\hline 6 & Флюорит & $\mathrm{CaF}_{2}$ & $0 \div 15$ & $<2.85$ \\
\hline 7 & Веберит & $\mathrm{Na}_{2} \mathrm{MgAlF}_{7}$ & $0 \div 6$ & $>2.5$ \\
\hline 8 & Нейборит & $\mathrm{NaMgF}_{3}$ & $2 \div 5$ & \\
\hline 9 & $\alpha-, \beta-, \gamma$ - глинозем & $\mathrm{Al}_{2} \mathrm{O}_{3}$ & & \\
\hline
\end{tabular}


характеристикам, пробам промышленного электролита стандартных образцов; эта процедура достаточно сложная, трудоемкая и на практике не всегда возможная.

Нами для автоматического анализа фазового состава образцов электролита алюминиевого производства предлагается бесстандартный , структурно-чувствительный $К Р Ф A$ по методу Ритвельда на основе разработанного многопопуляционного эволюционного алгоритма [2]. Так как большинство аналитических лабораторий обеспечены соответствующим оборудованием и возможностью проведения химического анализа, например, рентгеноспектральным флуоресцентным методом, то для повышения точности КРФА привлекается информация о количественном элементном составе образцов. Разработанный метод является гибким и может использоваться для контроля состава любых кристаллических систем на любом современном промышленном производстве.

\section{Экспериментальная часть}

Метод самоконфигурирования генетических алгоритмов

Полнопрофильный анализ по методу Ритвельда широко используется для лабораторного количественного рентгенофазового анализа. Однако его приложения для производственного технологического контроля недостаточно развиты. Причина в том, что метод Ритвельда основан на нелинейном методе наименьших квадратов (MHK), который для сходимости требует установки корректных исходных приближений уточняемых параметров для каждого образца. В лабораторном варианте метода требования к точности исходных приближений ниже, так как имеется возможность их интерактивного поэтапного уточнения. Но для технологического контроля требуется высокая степень автоматизма и способность унифицированного анализа большого количества образцов. При этом автоматическое уточнение большого числа профильных и структурных параметров из их унифицированных исходных значений, не достаточно хорошо подходящих одновременно ко всем образцам, часто приводит к расходимости МНК. Одним из вариантов решения этой задачи является применение генетических алгоритмов (ГА) для выбора начальных значений параметров образца, эволюционного отбора перспективных параметров и их дальнейшего автоматического уточнения по МНК метода Ритвельда.

Эффективность оптимизации при использовании эволюционных алгоритмов (в частности, ГА) зависит от выбора используемых генетических операторов: селекции, скрещивания, мутации и замещения. При этом оптимальные настройки алгоритма (обеспечивающие получение приемлемого результата за кратчайшее время) могут меняться при переходе от одной задачи к другой. Поэтому перспективны процедуры их динамической самоадаптации и самоконфигурирования, например, [3] и [4]. Суть метода самоконфигурирования состоит в том, что подбор оптимальных операторов производится из заданного множества их возможных вариантов самим алгоритмом в ходе решения задачи. Вероятности для операторов быть выбранными для порождения очередного потомка определяются, исходя из успешности этих операторов на предыдущих поколениях. Вероятность задействования на следующем поколении у лучшего оператора (того оператора, который позволяет получать лучшие решения задачи - лучшие параметры образца) повышается, у остальных - снижается, что приводит к автоматическому выбору наилучшего оператора путем увеличения вероятности его использования. Основные

$$
-265-
$$


этапы самоконфигурируемого генетического алгоритма (СГА) безусловной оптимизации могут быть описаны следующим образом.

1. При старте алгоритма выбор того или иного варианта для каждого вида оператора (селекция, скрещивание, мутация) является равновероятным (вероятность применения каждого варианта, например, селекции равна $p=1 / z$, где $\mathrm{z}-$ число операторов данного вида). То есть пока не накоплена статистика по эффективности применения, равновероятно применяются несколько вариантов селекции, несколько вариантов мутации, несколько вариантов скрещивания.

2. На каждом поколении оценка эффективности применения того или иного варианта операторов ГА производится по средней пригодности индивидов эволюции, получаемых с его помощью: average fitness $s_{i}=f_{i} / n_{i}, i=1,2, \ldots, z$, где average fitness $s_{i}-$ средняя пригодность порожденных потомков; $f_{i}$ - сумма пригодностей индивидов, полученных $i$-м оператором; $n_{i}$ - количество индивидов, полученных $i$-м оператором (данного вида, например, селекции); $z$ - число операторов.

3. При генерации следующего поколения вероятность использования наиболее эффективного варианта оператора увеличивается на $((z-1) \cdot K) /(z \cdot N)$, а вероятности всех остальных уменьшаются на $K /(z \cdot N)$, где $N$ - число поколений, $K$ - константа (равная 2 для рассматриваемых задач). В то же время вероятность ни одного из вариантов не может стать равной нулю (ниже заданного порога), сумма всех вероятностей для одного вида оператора всегда равна единице. При достижении заданного порога соответствующий вариант оператора перестает отдавать часть своей вероятности, а наиболее эффективный, соответственно, не получает ее. Таким образом, сохраняется возможность применения даже самых неэффективных операторов, так как на определенных этапах работы алгоритма эффективнее могут оказаться одни операторы, на других этапах - другие.

4. Для порождения каждого потомка следующего поколения стохастически (согласно полученным распределениям вероятностей) выбираются операторы, при помощи которых он и будет сгенерирован.

Самоконфигурирование освобождает пользователя от подбора настроек генетического алгоритма, при этом эффективность решения задачи остается приемлемой (при наилучшем выборе параметров генетического алгоритма эффективность решения задач несколько выше, но подбор параметров ГА требует времени и высокой квалификации пользователя).

Эффективность СГА может быть повышена при использовании его в рамках островной эволюционной (кооперативно-конкурирующей) модели, когда создаются несколько популяций, существующих отдельно друг от друга, лишь в некоторые моменты обменивающиеся генетическим материалом. Так обеспечивается более равномерное распределение возможных решений поставленной задачи по поисковому пространству. Поэтому для решения задачи количественного рентгенофазового анализа создана следующая реализация самоконфигурирования мультипопуляционного параллельного генетического алгоритма. В ней генерируется $n$ различных популяций из моделей определяемого вещества, и на каждом из $n$ вычислительных узлов многоядерного ПК запускается индивидуальный однопопуляционный СГА. В начале процесса генерируются случайные индивиды - наборы чисел, состоящие из значений уточняемых параметров метода Ритвельда для сгенерированных моделей, которые распределены по 
пространству поиска. На каждом из вычислительных узлов с помощью операторов рекомбинации и селекции эволюционно происходит формирование потомков с меньшими значениями целевой функции. Операторы мутации случайным образом «разбрасывают» их по пространству поиска, иногда с увеличением значения целевой функции. Часть моделей с меньшим значением целевой функции уточняются с помощью $M H K$ метода Ритвельда. Затем, в результате общей селекции формируется новая популяция тестовых моделей-потомков, в среднем, с лучшей пригодностью. Определенное количество лучших тестовых моделей из популяций на рабочих узлах отправляется на управляющий вычислительный узел СГА. Все эти решения, накопленные на управляющем узле, сортируются в порядке уменьшения значения целевой функции. Периодически часть лучших решений, накопленных к данному поколению эволюции на управляющем узле, случайно отбирается и рассылается обратно в популяции на рабочих узлах случайным образом. Такая умеренная миграция обеспечивает распространение удачных решений по популяциям и улучшает общую сходимость.

Самоконфигурирование реализовано для индивидуальных процессов $Г А$ на рабочих узлах согласно вышеописанной схеме СГА. Для каждого процесса задается стандартный набор генетических операторов - по несколько операторов каждого типа. В частности, одноточечное, двухточечное и равномерное скрещивание; ранговая и турнирная селекция с разными размерами турнира; низкая, средняя и высокая вероятность мутации. Распределение вероятностей для выбора конкретного типа оператора при генерации следующего поколения данной популяции постоянно смещается в пользу более эффективных вариантов операторов за счет менее эффективных. В данной реализации эффективность конкретных генетических операторов оценивается только локально, для индивидуальных ГА на отдельных рабочих узлах, без учета их эффективности в процессах ГА на других узлах. Последнее может являться способом повышения общей эффективности эволюционного КРФА, но требует изучения.

\section{Метод полнопрофильного КРФА в параллельном СГА}

КРФА по методу Ритвельда основан на итерационной минимизации с помощью нелинейного $M H К$ невязки между расчетным и экспериментальным профилями дифрактограммы:

$$
\Phi\left(\bar{P}_{k+1}\right)=\sum_{i} w_{i}\left(\operatorname{Yo}\left(2 \Theta_{i}\right)-Y c\left(\bar{P}_{k}+\Delta \bar{P}_{k}, 2 \Theta_{i}\right)\right)^{2} \rightarrow 0
$$

где $Y$, $Y c$ - экспериментальные и вычисленные интенсивности в точках профиля $2 \theta_{\mathrm{i}} ; w_{i}-$ весовые коэффициенты; $\bar{P}_{k}$ - вектор значений профильных, микроструктурных и кристаллоструктурных параметров, полученных на $k$-й итерации; $\Delta \bar{P}_{k}-$ приращения к ним, вычисляемые по МНК; при $k=0$ задаются исходные значения всех параметров.

Уточняемая по методу Ритвельда часть параметров вектора $\bar{P}$ определяет состав параметрических строк - индивидов эволюции по ГА. Полный набор параметров вектора $\bar{P}$, включая фиксированные и уточняемые, представляет тестовую модель характеристик многофазного образца. При выполнении эволюционного КРФА необходимо задавать область значений уточняемых параметров. Лучшие из значений, находимых в этой области в процессе эволюции по $Г A$, используются в качестве исходных значений для уточнения по методу Ритвельда. Для задания области поиска на параметры накладываются ограничения - для каждого параметра

$$
\text { - } 267 \text { - }
$$


задается интервал значений, который может устанавливаться в достаточно широких границах, перекрывающих их возможные вариации. Например, в качестве исходных значений структурных параметров используются кристаллические структуры фаз, выбранные из базы кристаллоструктурных данных в результате качественного РФА данного образца. При этом в качестве уточняемых структурных параметров могут задаваться координаты атомов, расположенных в общих кристаллографических позициях, а также коэффициенты их заполнения атомами, если данная фаза является твердым раствором. Соответствующие интервалы значений задают возможные окрестности, в которых могут варьироваться координаты этих атомов и коэффициентов заполнения.

Для автоматизации полнопрофильного КРФА важно то, что всегда известно целевое значение $R$-фактора, предварительно определяемое в результате декомпозиции профиля дифрактограммы без данных о кристаллической структуре фаз (обычно по методу ЛеБэйла [5]). После достижения одной из тестовых моделей значения $R$-фактора, близкого к целевому, выполняется ее финальное уточнение по методу Ритвельда и производится расчет концентрации фаз $C_{a}$ в образце. При отсутствии аморфной фазы для расчета используется нормированное выражение:

$$
C_{\alpha}=S_{\alpha} Z_{\alpha} M_{\alpha} V_{\alpha} / \sum_{j=1}^{N} S_{j} Z_{j} M_{j} V_{j},
$$

где $S_{a}$ - масштабный коэффициент расчетной дифрактограммы $Y c_{a}$ фазы $a$, который выбирается для ее оптимального соответствия экспериментальной дифрактограмме $Y o ; V_{a}, Z_{a}, M_{a}-$ объем ячейки, число формульных единиц в ячейке и молекулярная масса фазы $a ; N-$ число фаз многофазного образца.

В присутствии аморфной фазы используется вариант КРФА с внутренним стандартом [6].

Проблемой эволюционного КРФА может являться узость области поиска, в которой $R$-фактор изменяется недостаточно значительно, чтобы служить эффективным критерием пригодности для эволюционного поиска по ГА. Поэтому для усиления чувствительности целевой функции пригодности к $R$-фактору добавлена невязка между вычисленными из данных $K P \Phi A$ и измеренными значениями элементного состава:

$$
R_{p}=100 \cdot\left\{\left(1-w_{X A}\right) \cdot \sqrt{\frac{\sum_{i} w_{i} \cdot\left[Y o_{i}-Y c_{i}(\bar{P})\right]^{2}}{\sum_{i} w_{i} \cdot\left(Y o_{i}\right)^{2}}}+w_{X A} \cdot \frac{\sum_{t} w_{t} \cdot\left[\sum_{\alpha} p_{t \alpha} \cdot \frac{S_{\alpha} Z_{\alpha} M_{\alpha} V_{\alpha}}{\sum_{\alpha} S_{\alpha} Z_{\alpha} M_{\alpha} V_{\alpha}}-C_{t}^{X A}\right]^{2}}{\sum_{t} w_{t} \cdot\left(C_{t}^{X A}\right)^{2}}\right\},
$$

где $C_{t}^{X A}-$ измеренные значения элемента $t$ в образце; $P_{t a}-$ массовая доля химического элемента $t$ в фазе $a ; w_{X A}-$ весовой вклад в $R_{w p}$ данных о химическом составе образца (обычно 0,5 ).

Интеграция данной реализации полнопрофильного анализа по методу Ритвельда с параллельным СГА обеспечивает автоматическое выполнение КРФА. При этом целевой функцией СГА является профильный R-фактор метода Ритвельда.

Разработанный СГА был применен для анализа фазового состава и $К O$ комплекта из 24-х отраслевых стандартных образцов (OCO) электролита, к настоящему времени внедренного на пяти Российских алюминиевых заводах. Данные ОСО были выбраны по нескольким причи- 
нам: во-первых, они приготовлены из материала самого электролита нескольких алюминиевых заводов, т.е. отвечают составу и микроструктуре фаз реального промышленного электролита; во-вторых, ОСО аттестованы по химическому и фазовому составу с высоким качеством, стандартное отклонение данных аттестации составляет, в среднем 0.008 ед. КО; в-третьих, фазовый состав комплекта варьируется в широком интервале, например КО меняется от 1,9 до 3 [7].

Порошковые дифракционные данные получены с использованием $C u K_{\alpha}-$ излучения на дифрактометре Shimadzu-7000S. Образец растирался в агатовой ступке и подготавливался методом прямой набивки кюветы. Условия съемки: диапазон от 10 до $90^{\circ}$ по шкале $2 \theta$ с шагом $0,01^{\circ}, \Delta \mathrm{t}-$ 2,4 с, скоростью - 0,25\%мин. Структурные модели фаз брались из базы данных ICSD [8].

Метод СГА полностью автоматический, поэтому для анализа всех образцов использовался идентичный избыточный список фаз (см. табл. 1) и одинаковые настройки СГА. Для заданных фаз определялись значения параметров, указанные в табл. 2 (всего 90 параметров). Если фаза отсутствовала в образце, ее масштабный коэффициент обнулялся и концентрация, соответственно, тоже. Для каждого образца проводили по три запуска СГА и далее брали усредненные значения их фазовых концентраций. Для КРФА одного образца по программе СГА затрачивалось около 4-5 мин.

В аналитических лабораториях на алюминиевых заводах используются современные аналитические комплексы, совмещающие рентгеновскую дифракцию с рентгенофлуоресцентным анализом. Обычно это дифрактометры с фиксированными каналами на кальций и магний. Поэтому в химической части функции пригодности (4) использовались данные по содержанию именно этих двух элементов в образцах электролита.

Для работы СГА были выбраны следующие генетические операторы, вероятность использования которых адаптивно варьировалась в ходе эволюционного процесса при вычислении каждого образца:

Таблица 2. Уточняемые параметры по СГА для образцов электролита

Table 2. Refinable SGA parameters for electrolyte samples

\begin{tabular}{|c|c|}
\hline Фаза & Перечень уточняемых параметров \\
\hline \multicolumn{2}{|r|}{ Параметр коррекции сдвига нуля дифрактограммы } \\
\hline $\mathrm{Na}_{3} \mathrm{AlF}_{6}$ & $\begin{array}{l}S, a, b, c, \beta, U, W, E t a 0, A s y m l, \text { координаты атомов (12 позиций), текстура по } \\
\text { Марч-Далласу по направлению } h k l[112]\end{array}$ \\
\hline $\mathrm{Na}_{5} \mathrm{Al}_{3} \mathrm{~F}_{14}$ & $S, a, c, U, W, E t a 0, A s y m 1$, координаты атомов (9 позиций) \\
\hline $\mathrm{NaCaAlF}_{6}$ & $S, a, b, c, \beta, U, W$, Eta0, Asyml \\
\hline $\mathrm{Na}_{2} \mathrm{Ca}_{3} \mathrm{Al}_{2} \mathrm{~F}_{14}$ & $S, a, U, W$, Eta0, Asyml \\
\hline $\mathrm{CaF}_{2}$ & $S, a, U, W$, Eta0, Asym1 \\
\hline $\mathrm{Na}_{2} \mathrm{MgAlF}_{7}$ & $S, a, b, c, U, W$, Eta0, Asyml \\
\hline $\mathrm{NaMgF}_{3}$ & $S, a, b, c, U, W$, Eta0, Asyml \\
\hline$\alpha-\mathrm{Al}_{2} \mathrm{O}_{3}$ & $S, a, b, U, W$, Eta0, Asyml \\
\hline$\gamma-\mathrm{Al}_{2} \mathrm{O}_{3}$ & $S, a, U, W$, Eta0, Asyml \\
\hline
\end{tabular}

$S$ - масштабный коэффициент фазы; $a, b, c, \beta$ - параметры кристаллической ячейки; $U, W$ - параметры полуширины пика (по модели псевдо-Войгта); Eta0 и Asym1- параметры формы пика и асимметрии соответственно. 
- операторы селекции: турнирная с размером турнира 3, 5, 7, 9, ранговая;

- операторы скрещивания: двухточечное, трехточечное, равномерное;

- операторы мутации: низкий уровень, средний уровень, высокий уровень, с тремя значениями среднеквадратического отклонения каждый.

Кроме этого, была применена гибридизация генетического алгоритма с локальным спуском по Ламарку каждые 10 поколений [9].

\section{Результаты и обсуждения}

В качестве примера итоговых результатов, получаемых при полнопрофильном КРФА по параллельному $C Г А$, на рис. 1 представлен типичный результат моделирования дифрактограммы одного из образцов электролита. Профильный $R$-фактор составил 8,6 \% отн.

На рис. 2 приведен типичный график сходимости, полученный в процессе анализа по СГА этого же образца. По оси абсцисс указаны номера поколений эволюции (нулевой номер - начальная случайная генерация всех популяций), по оси ординат - соответствующие значения функции пригодности для лучшего решения из числа накопленных на управляющем ядре. Мы видим, что решение найдено приблизительно на 80-м поколении.

На рис. 3 сравниваются аттестованные значения $K O$ с $K O$, рассчитанными из фазового состава, полученного по СГА. График в идеале должен проходить через нуль под углом $45^{\circ}$, а отклонения от него будут свидетельствовать о наличии систематической межметодической погрешности. Для количественной оценки ее существования на рис. 3 представлено также регрессионное линейное уравнение $(y=a+b x)$. Соответственно, стандартное отклонение $S D$ показывает величину случайной межметодической погрешности. Эти расчеты и график выполнены с помощью программы статистической обработки аналитических данных ORIGIN.

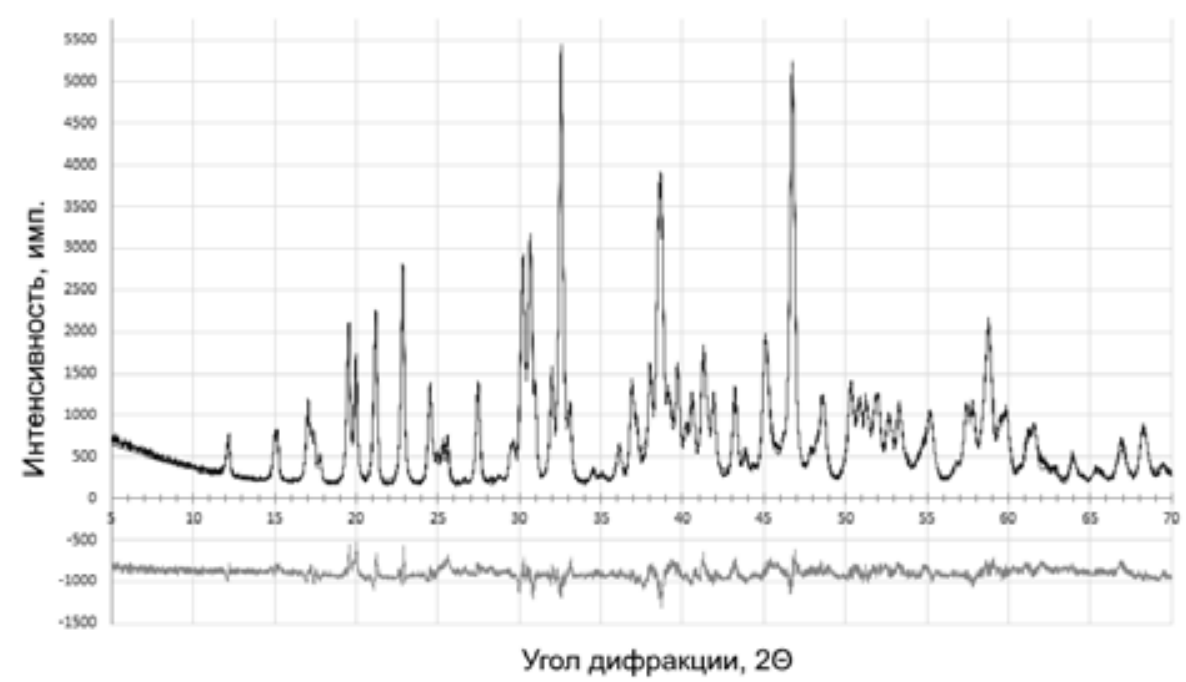

Рис. 1. Результаты моделирования дифрактограммы одного из образцов электролита по параллельному СГА с разностной кривой

Fig. 1. Model powder pattern calculated by the parallel SGA for an electrolyte sample and the difference curve 


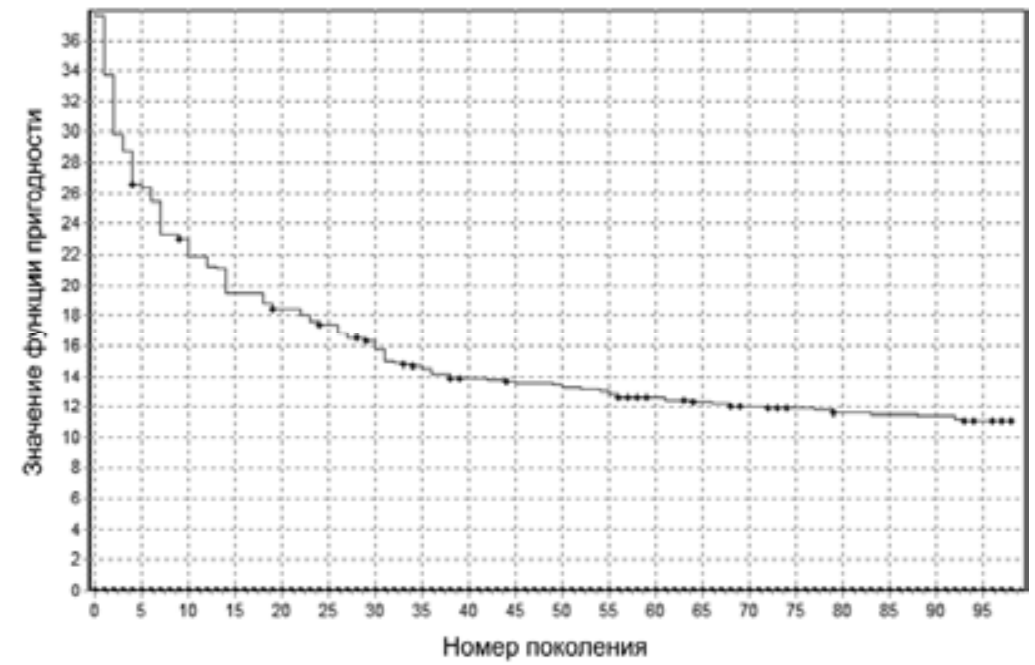

Рис. 2. Типичный график сходимости анализа образца электролита по $С Г А$

Fig. 2. A typical SGA convergence graph obtained during the analysis of an electrolyte sample

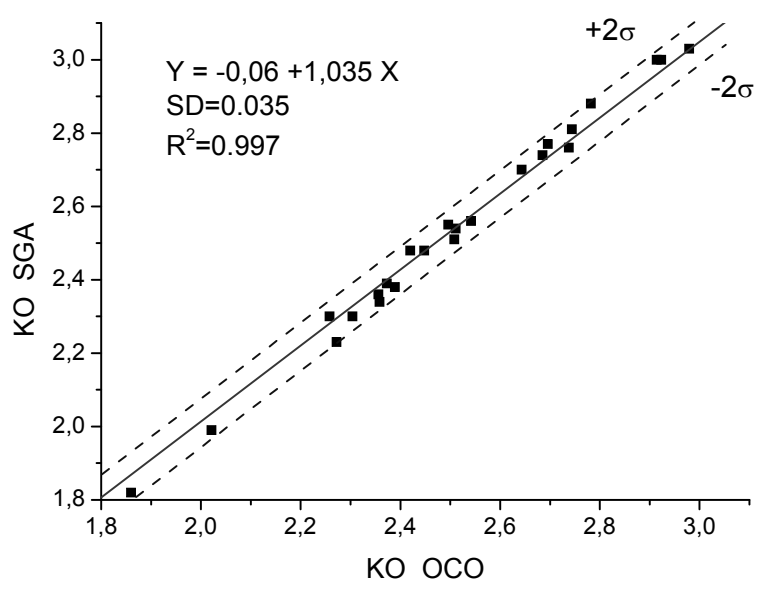

Рис. 3. График соответствия расчетных и аттестованных $K O$ отраслевых стандартных образцов электролита (КО ОСО - значения аттестованных $K O$, KO SGA - значения $K O$, рассчитанных из фазового состава по СГА; SD - стандартное отклонение (\% масс.); $\mathrm{R}$ - коэффициент корреляции; регрессионное уравнение $y=a+b x)$

Fig. 3. Correspondence between the calculated and certified values of $\mathrm{CR}$ for branch reference samples of the electrolyte (KO OCO - certified values of CR, KO SGA - values of CR calculated from the phase composition in $\mathrm{SGA}, \mathrm{SD}-$ standard deviation ( $\%$ mass), $\mathrm{R}$ - correlation coefficient, regression equation $\mathrm{y}=\mathrm{a}+\mathrm{bx}$ )

\section{Выводы}

1. Определенные по $С Г А$ значения $K O$ соответствуют аттестованным значениям с точностью, характеризуемой стандартным отклонением 0,035 ед. КО и укладываются в 95\%-й интервал надежности, изображенный пунктиром на рис. 3.

2. Регрессионное уравнение хорошо соответствует уравнению $y=a+b x$, при этом коэффициент $a$ статистически незначимо отличен от 0 , но $b$ завышен на 3,5 \% отн. Последнее показывает, что имеет место небольшое систематическое завышение результатов 
СГА. Это вызвано завышением концентрации фазы криолита $N a_{3} A l F_{6}$ и свидетельствует о неполноте автоматического уточнения ее кристаллической структуры по СГА, искажаемой вследствие неполного перехода высокотемпературной модификации криолита в низкотемпературную при неравновесной кристаллизации проб электролита в процессе пробоотбора на заводах.

3. В целом, полученные характеристики соответствуют технологическим требованиям, предъявляемым на алюминиевом производстве к точности анализа КО, и метод СГА может быть рекомендован к внедрению для оперативного контроля КО электролита после устранения причин отмеченной систематической погрешности.

Исследование выполнено при поддержке Российского фонда фундаментальных исследований, Правительства Красноярского края, Красноярского краевого фонда поддержки научной и научно-технической деятельности в рамках проекта № 16-43-243049.

\section{Список литературы}

1. Apisarov A., Frolov A., Gusev A. et al. Modiefid alumina-cryolite bath with high electrical conductivity and dissolution rate of alumina. Light metals 2007. P. 571-576.

2. Zaloga A., Akhmedova S., Yakimov I., Burakov S., Semenkin E., Dubinin P., Piksina O., Andryushchenko E. Genetic Algorithm for Automated X-Ray Diffraction Full-Profile Analysis of Electrolyte Composition on Aluminium Smelters. Lecture Notes in Electrical Engineering 2016. Vol. 383, P. 79-93.

3. Semenkin E.S., Semenkina M.E. The Choice of Spacecrafts' Control Systems Effective Variants with Self-Configuring Genetic Algorithm. In: Ferrier, J.L. et al (Eds.): Informatics in Control, Automation and Robotics: Proceedings of the 9th International Conference ICINCO'2012. Rome: Italy, 2012. Vol. 1, P. 84-93.

4. Semenkin E.S., Semenkina M.E. Self-configuring Genetic Algorithm with Modified Uniform Crossover Operator. Advances in Swarm Intelligence. Lecture Notes in Computer Science 7331. Springer-Verlag, Berlin Heidelberg 2012. P. 414-421.

5. Le Bail A. The Profile of a Bragg Reflection for Extracting Intensities. Chapter 5. Powder Diffraction Theory and Practice, ed. R.E. Dinnebier and S.J.L. Billinge. Cambridge, Royal Society of Chemistry, 2008. 571 p.

6. Bish D.L., Howard S.A. Quantitative phase analysis using the Rietveld method. Journal of Applied Crystallography 1998, Vol. 21, P. 86-91.

7. Якимов И.С., Дубинин П.С., Залога А.Н., Пиксина О.Е., Кирик С.Д. Разработка отраслевых стандартных образцов электролита алюминиевых электролизеров. Стандартные образиы, 2008 (4), C. 30-42 [Yakimov I.S., Dubinin P.S., Zaloga A.N., Piksina O.E., Kirik S.D. Development of industry standard electrolyte samples of aluminum electrolyzers. Certified Reference Materials 2008, Vol. 4, P. 30-42. (In Russ.)]

8. Inorganic Crystal Structure Database (ICSD) http://www2.fiz-karlsruhe.de/icsd_publications. html.

9. M. Tim Jones. AI Application Programming (Programming Series). Charles River Media, 2003. $363 \mathrm{p}$. 